\title{
Mental Health Issues and Pervasive Computing
}

\author{
David Haniff \\ ActivityComputing.com \\ Oldbrook, Milton Keynes, MK6 2XT \\ +44 (0)1908236379 \\ davejhaniff@hotmail.com
}

\begin{abstract}
This poster describes work being undertaken in the use of pervasive computing for the treatment of mental health problems. The use of technology to help patients with psychological issues such as depression are explored and preliminary investigations are discussed.
\end{abstract}

\section{Categories and Subject Descriptors}

J.3.3 [Life and Medical Sciences], H.5.2 [User Interfaces]

\section{General Terms}

Design, Human Factors.

\section{Keywords}

Mental Health, Pervasive Computing, Ubiquitous Computing.

\section{INTRODUCTION}

This poster presents preliminary investigations into the issue of pervasive computing and mental health. Research has been carried out on the use of computers to diagnose and provide aid to patients suffering from mental illness [5], as well as providing tasks for patients outside of psychotherapy sessions [7]. Mental health problems can be defined as disorders within the mind that effect the normal life of an individual. One in four people suffer from a mental health problem within a year, severe mental health problems are, however more rare. The problems can be crippling for the person but is often neglected because it is within the mind and not a physical problem. They can be distressing for the individual and for those around them. There are a range of treatments for various mental illnesses such as medication, electric shock treatment and cognitive therapy. Pervasive computing rather than replacing these treatments can be used to support the individual with the problem. The aims for the research are to investigate how pervasive technology can support clinical psychologists in the treatment of mental illness, to examine the type of information that is appropriate for the patient with the mental illness and to build and evaluate the use of pervasive technology for mental illness. There are many types of mental illness suffered by patients, however, the illnesses addressed by this research are those where cognitive therapy can be useful, such as depression, and Obsessive Compulsive Disorder (OCD).

\section{DEPRESSION}

(C)David Haniff, 2007

Published by the British Computer Society

Volume 2 Proceedings of the 21st BCS HCI Group Conference

HCI 2007, Lancaster University, 3-7 September 2007

Devina Ramduny-Ellis \& Dorothy Rachovides (Editors)
Depression is a state of mind where the individual feels low which can be caused by negative thoughts. Depression can have a profound effect on people's lives leading to symptoms ranging from lethargy to some in extreme cases to commit suicide. There are a number of treatments for depression such as anti-depressants, for example, Prozac, which is intended to lift the mood of the patient. Cognitive therapy can also be used to alter the way the patient thinks. Negative thoughts that can cause depression can be replaced by positive thoughts or challenged by the patient. Negative thoughts for some people are caused by seeing an insurmountable problem and having a feeling of hopelessness, they can be taught to break down a problem into smaller components and rewarding themselves when the smaller problem is solved. The causes of the depression need to be identified by the patient, the thoughts that trigger this emotion can then be worked upon. Another method for combating depression is to structure the day with activities that lift the patient's mood, for example, chatting to a friend or doing exercise. Furthermore, depression can also be helped by the use of physical exercise which can release Serotonin into the brain. This helps to relax the mind and can lift the mood of the patient.

Pervasive computing can aid the patient by providing reminders about activities that are beneficial to the mood of the patient. This can take the form of a Personal Digital Assistant (PDA) and voice output gently reminding the patient about tasks that they should be doing through out the day. Technology can also be used to identify particular thoughts through the patient verbalising a key word and presenting a challenge to that thought when it occurs. The computer can use speech recognition to identify a particular word associated with the thought and the challenge to the thought presented by the computer can be decided in co-operation with a cognitive therapist. These types of applications will be further explored by the research.

With regard to exercise, an exercise routine can be presented to the user through a PDA and when targets have been met these can be ticked off on the computer, having a structured routine will help their mental and physical health. The software could calculate the routine for them, setting small targets initially in order to gain a sense of achievement. This routine has to be agreed by their doctor. Furthermore, technology can be used to remind people when to do exercise [2]. In addition, wearable computers have been used for sports training, providing feedback on their performance [4], this can be used as a personal training device for the patient. Words of encouragement can be presented to the user from the system when personal goals have been achieved, this will encourage the patient and give them confidence which may lift their mood further.

In addition, reminders of people in the patients life can lift their mood, for example their wives or children. A PDA can be used to provide images, speech and video to distract the patient and help them think about the things that are important in their 
lives. Figure 1 shows an example of an application which was written in Java 2. When the patient is low these media files can be used to trigger positive thoughts and lift their mood.

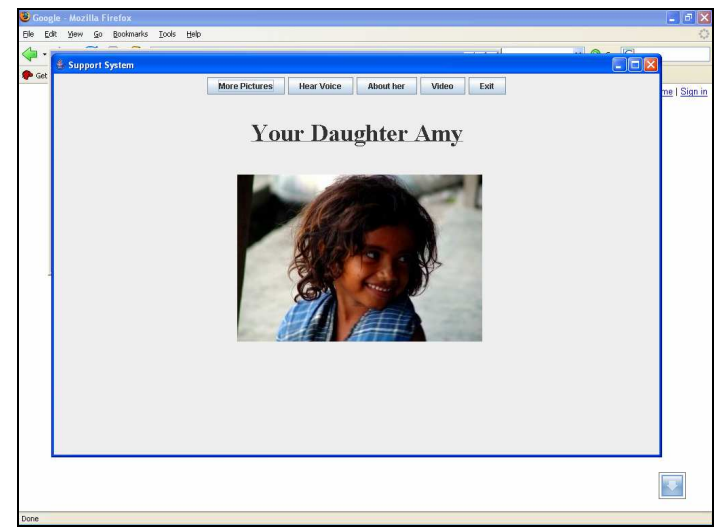

Figure 1: An Application that presents pictures, voice, words and video of a patients daughter.

\section{OBSESSIVE COMPULSIVE DISORDER}

Obsessive Compulsive Disorder (OCD) is a mental illness that causes the sufferer to mentally or physically repeat irrational actions, internally or physically. "Obsessive compulsive disorder (OCD) is a common mental health condition that affects $2 \%$ of the population." [6]. To the patient these actions can be seen as completely rational, what separates these actions from 'normal' actions is the culture in which the person is nurtured. There are rules and conventions that determine what is appropriate human behaviour. The treatment for such a condition has been conventionally through medication and/or cognitive therapy. Certain anti-depressants are credited to ease this illness by acting on the repetitive nature of this action. The cognitive therapy indicates appropriate techniques and mental actions to reduce or cure the occurrence of this inappropriate behaviour.

Using an analogy with computer programming the internal processing of information is akin to the execution of a 'while' loop without a condition stopping the repetitive loop from its process. For example, there are many cases of OCD patients who wash their hands constantly without due course. The sufferers wash their hands to an extent that the skin becomes raw when they know that this detrimental to their person. The mind is caught in a loop without rational thought to stop the action. There have been cases whereby the sufferer performs a certain ritual that certifies their well being even though there is no causal relationship between performing the act and the consequences of the act. For instance, repeating certain words to ensure a loved ones safety.

This poster suggests that the treatment of this mental illness by the use of technology embedded within the environment can prove fruitful. The technology adapts in a unique way whereby ubiquitous computing responds to the mental state of the user. As with a pace-maker, technology is used to aid the patient but instead of being solely a personal device the technology is distributed within the environment too. Wearable computers and external sensors have been used for the visually impaired, there has however not been widespread adoption of this technology for those with various needs. This project hopes to stimulate discussion within the HCI community about the use of technology and in particular ubiquitous computing due to its pervasiveness and adaptability to situations to treat mental illness. OCD often manifests itself in reaction to situations that the individual might find them self in, pervasive computing can be situationally-aware (using sensors to provide contextual information) and can therefore provide the flexibility required by the sufferer. For some sufferers the condition worsens under stress, a state of anxiety can be detected through various onbody sensors and appropriate information can be given to counteract the anxiety. A particular object or situation can trigger anxiety, pervasive computing can sense these objects and situations and provide help for the sufferer, for instance RF tags could be used to identify disturbing objects to the patient. The help could well be auditory, for example, words of comfort or instructions on what to do next may be spoken to the user. Sensors have been used in medical applications, for example, Kientz and Abowd [3] describe the use of toy-embedded sensors to collect data concerning developmental milestones in the early detection of autism in children. Ebrahimi et al. [1] describe brain computer interfaces whereby signals from the brain can control external devices. One possible way of detecting OCD is to monitor brain patterns and a computing device can therefore respond appropriately.

The pervasive systems developed can be integrated with cognitive therapy which in many ways attempts to change the way someone thinks in order to relieve the individual from the symptoms of the condition. In some cases a cure may be achieved as certain actions become automatic, however, the potential for this technology to rid the sufferers of this illness needs to be explored.

\section{CONCLUSION}

The research described within this poster aims to examine and provide technology based solutions to the increasing quantity of the population effected by mental illness. The illnesses to be investigated by this research are depression and obsessive compulsive disorder. The research is very much at an early stage and can potentially greatly influence the lives of people suffering from mental problems.

\section{REFERENCES}

[1] Ebrahimi, T., Vesin, J. \& Garcia, G. (2003) Brain Computer Interface in Multimedia Communication. IEEE Signal Processing Magazine, February 2003.

[2] Fogg, B. (2002) Persuasive Computing. Morgan Kaufm.

[3] Kientz, J.A., Abowd, G.D. (2006) Designing Technology to Aid in the Early Detection of Developmental Delay in Children. UbiHealth 2006.

[4] Knight, J. F., Schwirtz, A., Psomadelis, F., Baber, C., Bristow, H. W., Arvanitis, T. N. (2005) The Design of Sunvest. Personal and Ubiquitous Computing, 9 (1) 6-19.

[5] Marks, I. (1999) Computer Aids to Mental Health Care, Canadian Journal of Psychiatry, 44, 548-555.

[6] NHS Direct. (2007) http://www.nhsdirect.nhs.uk/articles/ article. aspx? articleID $=266$.

[7] Sa, M. D., Carrico, L., Antunes, P. (2007) Ubiquitous Psychotherapy, IEEE Pervasive Computing, 6(1), 20-27. 